\title{
Development and Internal Validation of Supervised Machine Learning Algorithms for Predicting the Risk of Surgical Site Infection Following Minimally Invasive Transforaminal Lumbar Interbody Fusion Under Quadrant Channel
}

Haosheng Wang

Taizhou Central University

Tingting Fan

Baoji City Hospital of Traditional Chinese Medicine

Bo Yang

Baoji City Hospital of Traditional Chinese Medicine

Qiang Lin

Baoji City Hospital of Traditional Chinese Medicine

Wenle Li

Xiangyang Central Hospital

Mingyu Yang ( $D$ 616353108@qq.com )

Taizhou Central Hospital

\section{Research article}

Keywords: Surgical infection sites, Machine learning, Risk factors, minimally invasive transforaminal lumbar interbody fusion, prediction model

Posted Date: September 20th, 2021

DOI: https://doi.org/10.21203/rs.3.rs-882632/v1

License: (c) (i) This work is licensed under a Creative Commons Attribution 4.0 International License.

Read Full License 


\section{Abstract}

Background: Machine Learning $(\mathrm{ML})$ is rapidly growing in capability and is increasingly applied to model outcomes and complications in medicine. Surgical site infections (SSI) are a common postoperative complication in spinal surgery. This study aimed to develop and validate supervised ML algorithms for predicting the risk of SSI following minimally invasive transforaminal lumbar interbody fusion (MIS-TLIF) under the Quadrant channel.

Methods: This single-central retrospective study included a total of 705 cases between May 2012 and October 2019. Data of patients who underwent MIS-TLIF under the Quadrant channel was extracted by the electronic medical record system. The patient's clinical characteristics, surgery-related parameters, and routine laboratory tests were collected. Univariate and multivariate logistic regression analyses were used to screen and identify independent risk factors for SSI. Then, the independent risk factors were imported into six ML algorithms, including k-Nearest Neighbor (KNN), Decision Tree (DT), Support Vector Machine (SVM), Random Forest (RF), Multi-Layer Perceptron (MLP), and Naïve Bayes (NB), to develop a prediction model for predicting the risk of SSI following MIS-TLIF under Quadrant channel. During the training process, 10 -fold cross-validation was used for validation. Indices like the area under the receiver operating characteristic (AUC), sensitivity, specificity, and accuracy (ACC) were reported to test the performance of ML models.

Results: Among the 705 patients, SSI occurred in 33 patients (4.68\%). The univariate and multivariate logistic regression analyses showed that preoperative glycated hemoglobin A1c ( $\mathrm{HbA1c})$, estimated blood loss (EBL), preoperative albumin, body mass index (BMI), and age were all independent predictors of SSI. In predicting SSI, six ML models posted an average AUC of $0.60-0.80$ and an ACC of $0.80-0.95$, with the NB model standing out, registering an average AUC and an ACC of 0.78 and 0.90 . Then, the feature importance of the NB model was reported.

Conclusions: $\mathrm{ML}$ algorithms are impressive tools in clinical decision-making, which can achieve satisfactory prediction of SSI with the NB model performing the best. The NB model may help access the risk of SSI following MIS-TLIF under the Quadrant channel and facilitate clinical decision-making. However, future external validation is needed.

\section{Background}

Minimally invasive transforaminal lumbar interbody fusion (MIS-TLIF) is a classic minimally invasive operation for the treatment of lumbar degenerative diseases such as lumbar disc herniation, lumbar spinal stenosis, and lumbar spondylolisthesis. The operation uses channels to complete decompression and bone graft fusion and fixation. Compared with the traditional open TLIF, it better retains the paravertebral muscle structure, less intraoperative bleeding and faster postoperative recovery[1-3]. It has become the mainstream operation scheme for minimally invasive fusion surgery at present. 
Although surgical site infections (SSI) following MIS-TLIF are lower than those following open TLIF, previous literature has reported that the incidence of SSI after MIS-TLIF is not uncommon as this procedure is widely used in the clinic $[4,5]$. Surprisingly, over $20 \%$ of the patients who were re-admitted within 30 days after operation were caused by SSI[6,7]. Meanwhile, the rise in the number of spinal surgeries results in an expanding number of SSI, which poses a critical challenge to patients and clinicians. At present, there are studies on the analysis of preoperative and intraoperative risk factors for SSI after total spinal open surgery, but there is little literature investigating the risk factors for SSI following MIS-TLIF under the Quadrant channel. Previously, most studies only described these risk factors as relative risks (RR) or odds ratios $(\mathrm{OR})[7,8]$, which are not sufficient to evaluate the risk of SSI following MIS-TLIF under the Quadrant channel. Therefore, a comprehensive prediction model combining risk factors is needed to assess SSI risk for patients. Identification of high-risk surgical populations might help target interventions to patients at high risk, reduce the risk of hospitalization, and improve the clinical outcome.

Machine learning $(\mathrm{ML})$ is a form of artificial intelligence (Al) in which algorithms automatically learn and improve by identifying patterns and complex relationships, with the ultimate goal of making decisions using minimal human intervention[9-13]. Through the good performance exhibited by ML algorithms in medical big data, we have the potential to obtain more superior prediction tools than traditional statistical modeling modalities under certain conditions for better prediction of the risk of SSI. It is frustrating that there are currently very few studies training ML algorithms to predict SSI risk. As such, this study aimed to develop and validate ML-based models using preoperative and intraoperative variables to predict the risk of SSI following MIS-TLIF under the Quadrant channel.

\section{Methods}

\subsection{Patients}

The study was approved by the institutional review board (IRB) of Taizhou Central Hospital and was performed following national and international guidelines. The ethics committee waived the requirement for informed consent due to the retrospective nature of the study. A total of 705 patients, referred from May 2012 to October 2019 to the Department of Orthopedics, Taizhou Central Hospital (Taizhou, China) for MIS-TLIF owing to lumbar spine diseases, were enrolled in this research. The selection criteria were as follows: (1) age $\geq 18$ years; (2) diagnosis of lumbar degenerative disease, including lumbar disc herniation, lumbar spinal stenosis, lumbar spondylolisthesis, and lumbar instability; (3) undergoing single-or multi-segment MIS-TLIF under Quadrant channel. The exclusion criteria were as follows: (1) Patients were preoperatively complicated by active infection in the spine or other parts of the body, cauda equina syndrome, spinal deformity, and tumors. (2) Emergency surgery.

All operations were done by 4 experienced spinal surgeons at our institution. Patients were followed for a minimum of 1 year after the index operation to monitor all complications and incidences of revision surgery. All complications were recorded by the available electronic medical system. The primary 
outcome of interest was the occurrence of SSI. The diagnosis of SSI was based on the CDC criteria (Centre for Disease Control and Prevention)[14]. The primary diagnostic criteria for SSI were as follows. (1) the surgical wound showed redness, swelling, heat, pain, wave sense or combined with abscess, and drooling(2) Bacterial culture of wound exudate was positive (3) Patients with re debridement surgery, positive intraoperative lavage fluid or tissue bacterial culture (4) Laboratory tests for blood routine, Creactive protein, procalcitonin, as well as MRI examination or histopathological examination were performed to confirm SSI.

\subsection{Data collection}

The personal and medical information of patients, including age, body mass index (BMI), smoking, alcohol, chronic heart failure (CHF), diabetes, COPD, stroke, rheumatic disease, and diagnosis. The preoperative laboratory examinations, including blood routine examination, glycated hemoglobin A1c ( $\mathrm{HbA1c}$ ), liver function test, serum potassium, and serum calcium, were collected from the electronic medical record (EMR) system. Meanwhile, we recorded the surgery-related parameters, including operation time, intraoperative blood loss, surgical level, and the number of fusion segments in this investigation.

\subsection{Statistical analysis}

The statistical analyses were performed using SPSS for Windows software (ver. 26.0; SPSS Inc., Chicago, IL, USA). For continuous, normally distributed variables, means were calculated and compared using the independent student $t$ test; otherwise, the Mann-Whitney $U$ test was used for group comparisons. The chi-square test was used to analyze qualitative variables. Values of $\mathrm{P}<0.05$ were considered significant.

\subsection{Training and evaluation of ML models}

Forward step-wise multivariable logistic regression analysis, including covariates identified in univariate logistic analysis, was used to identify independent factors associated with SSI following MIS-TLIF under Quadrant channel. Meanwhile, the odds ratio (OR) and 95\% confidence intervals (Cls) were reported.

In its most basic sense, machine learning uses programmed algorithms that learn and optimize their operations by analyzing input data to make predictions within an acceptable range[15-18]. In this study, 10 -fold cross-validation was adopted, which means patients were randomly divided into a training set and a validation set at a ratio of 9:1 in each round. After 10 rounds, where all the patients are used for the test, the evaluation process ends. We developed five types of $\mathrm{ML}$ algorithms to model our data: k-Nearest Neighbor (KNN), Decision tree (DT), Support vector machine (SVM), Random Forest (RF), Multi-Layer Perceptron (MLP), Naïve Bayes (NB). When training the prediction model, we used 10-fold crossvalidation $(\mathrm{CV})$ on the training data to avoid overfitting and find the optimal hyperparameters. Then, Python software was used to further train the ML algorithms. To assess the performance of the $\mathrm{ML}$ algorithms, the area under the receiver operating characteristic (AUC) value, and sensitivity, specificity, the whole dataset accuracy (ACC), and confusion matrix of $\mathrm{ML}$ algorithms were reported. Of these, the ACC was determined by the following formula: 


$$
\begin{gathered}
A C C=\frac{T P+T N}{T P+T N+F P+F N} \\
\text { Sensitivity }=\frac{T P}{T P+F N} \\
\text { Specificity }=\frac{T N}{F P+T N}
\end{gathered}
$$

In these formulas, TP: true positive; TN: true negative; FP: false positive; and FN: false negative. When we evaluated the performance of different $M L$ algorithms, the closer the AUC was to 1, the better the classification performance of the model. In general, AUC $>0.7$ indicated good performance of the model. We next determined the AUC values by comparing different $\mathrm{ML}$ algorithms, with the best performing algorithm as the final predictive model. Data analysis was performed using programs written in Python programming. The Python packages, including Scikit-learn 0.24.2[19] and Python 3.8, are adopted for ML algorithms. A two-tailed $P<0.05$ was deemed statistically significant. The flowchart of the study is presented in Fig. 1.

\section{Results}

\subsection{Patients' characteristics}

In this study, a total of 705 cases were included, 401 (56.9\%) males and 304 (43.1\%) females, mean (58.7 \pm 7.2$)$ years old. Among the 705 cases, SSI occurred in 33 (4.68\%) cases, i.e., the SSI group; other cases were in the Non-SSI group. Among the SSI and Non-SSI groups, there were statistically significant differences in age $(p<0.001)$, BMI $(p<0.001)$, smoking $(p=0.002)$, diabetes $(p<0.001)$, ASA $(p=0.005)$, operation time $(p<0.001)$, estimated blood loss (EBL) $(p<0.001)$, number of fusion segments $(p<0.001)$, preoperative HbA1c $(p<0.001)$, preoperative albumin $(p<0.001)$, and preoperative fibrinogen $(p<0.001)$. There were no significant differences in remaining parameters between the SSI and Non-SSI groups. The detailed results of the parameters are shown in Table 1. 
Table 1

Comparison of variables between the SSI group and Non-SSI group

\begin{tabular}{|c|c|c|c|c|c|}
\hline & & Total & $\begin{array}{l}\text { Non- } \\
\text { SSI }\end{array}$ & SSI & $\begin{array}{l}P \\
\text { value }\end{array}$ \\
\hline \multicolumn{2}{|l|}{$\begin{array}{l}\text { Number of } \\
\text { patients }\end{array}$} & 705 & 672 & 33 & \\
\hline \multicolumn{2}{|l|}{ Age (years) } & $\begin{array}{l}58.7 \\
(7.2)\end{array}$ & $\begin{array}{l}58.4 \\
(7.1)\end{array}$ & $\begin{array}{l}65.0 \\
(6.5)\end{array}$ & $\begin{array}{l}<.001 \\
0.001\end{array}$ \\
\hline \multicolumn{6}{|l|}{ Sex (\%) } \\
\hline & Female & $\begin{array}{l}304 \\
(43.1)\end{array}$ & $\begin{array}{l}289 \\
(43.0)\end{array}$ & $\begin{array}{l}15 \\
(45.5)\end{array}$ & 0.858 \\
\hline & Male & $\begin{array}{l}401 \\
(56.9)\end{array}$ & $\begin{array}{l}383 \\
(57.0)\end{array}$ & $\begin{array}{l}18 \\
(54.5)\end{array}$ & \\
\hline \multicolumn{2}{|l|}{ BMI $\left(k g / m^{2}\right)$} & $\begin{array}{l}25.2 \\
(3.0)\end{array}$ & $\begin{array}{l}25.0 \\
(3.0)\end{array}$ & $\begin{array}{l}28.4 \\
(2.7)\end{array}$ & $\begin{array}{l}< \\
0.001\end{array}$ \\
\hline \multicolumn{6}{|l|}{ Smoking (\%) } \\
\hline & No & $\begin{array}{l}519 \\
(73.6)\end{array}$ & $\begin{array}{l}503 \\
(74.9)\end{array}$ & $\begin{array}{l}16 \\
(48.5)\end{array}$ & 0.002 \\
\hline & Yes & $\begin{array}{l}186 \\
(26.4)\end{array}$ & $\begin{array}{l}169 \\
(25.1)\end{array}$ & $\begin{array}{l}17 \\
(51.5)\end{array}$ & \\
\hline \multicolumn{6}{|l|}{ Alcohol (\%) } \\
\hline & No & $\begin{array}{l}479 \\
(67.9)\end{array}$ & $\begin{array}{l}457 \\
(68.0)\end{array}$ & $\begin{array}{l}22 \\
(66.7)\end{array}$ & 0.851 \\
\hline & Yes & $\begin{array}{l}226 \\
(32.1)\end{array}$ & $\begin{array}{l}215 \\
(32.0)\end{array}$ & $\begin{array}{l}11 \\
(33.3)\end{array}$ & \\
\hline \multicolumn{6}{|l|}{ Diabetes (\%) } \\
\hline & No & $\begin{array}{l}584 \\
(82.8)\end{array}$ & $\begin{array}{l}566 \\
(84.2)\end{array}$ & $\begin{array}{l}18 \\
(54.5)\end{array}$ & $\begin{array}{l}< \\
0.001\end{array}$ \\
\hline & Yes & $\begin{array}{l}121 \\
(17.2)\end{array}$ & $\begin{array}{l}106 \\
(15.8)\end{array}$ & $\begin{array}{l}15 \\
(45.5)\end{array}$ & \\
\hline \multicolumn{6}{|l|}{ COPD (\%) } \\
\hline & No & $\begin{array}{l}680 \\
(96.5)\end{array}$ & $\begin{array}{l}648 \\
(96.4)\end{array}$ & $\begin{array}{l}32 \\
(97.0)\end{array}$ & 1 \\
\hline & Yes & $25(3.5)$ & $24(3.6)$ & $1(3.0)$ & \\
\hline
\end{tabular}

Abbreviations: $\mathrm{BMI}$, body mass index; $\mathrm{COPD}$, chronic obstructive pulmoriary disease; $\mathrm{CHF}$, chronic heart failure; ASA, American Society of Anesthesiologists; EBL, estimated blood loss; HbA1c, glycated hemoglobin A1c; Alb, albumin; RBC, red blood cells; WBC, white blood cells; PT, prothrombin time; APTT: activated partial thromboplastin time; Fib, fibrinogen. 


\begin{tabular}{|c|c|c|c|c|c|}
\hline & & Total & $\begin{array}{l}\text { Non- } \\
\text { SSI }\end{array}$ & SSI & $\begin{array}{l}P \\
\text { value }\end{array}$ \\
\hline \multicolumn{6}{|c|}{ Stroke (\%) } \\
\hline & No & $\begin{array}{l}697 \\
(98.9)\end{array}$ & $\begin{array}{l}664 \\
(98.8)\end{array}$ & $\begin{array}{l}33 \\
(100.0)\end{array}$ & 1 \\
\hline & Yes & $8(1.1)$ & $8(1.2)$ & $0(0.0)$ & \\
\hline \multicolumn{6}{|c|}{$\begin{array}{l}\text { Rheumatic } \\
\text { disease (\%) }\end{array}$} \\
\hline & No & $\begin{array}{l}687 \\
(97.4)\end{array}$ & $\begin{array}{l}655 \\
(97.5)\end{array}$ & $\begin{array}{l}32 \\
(97.0)\end{array}$ & 0.583 \\
\hline & Yes & $18(2.6)$ & $17(2.5)$ & $1(3.0)$ & \\
\hline \multicolumn{6}{|l|}{ CHF (\%) } \\
\hline & No & $\begin{array}{l}702 \\
(99.6)\end{array}$ & $\begin{array}{l}669 \\
(99.6)\end{array}$ & $\begin{array}{l}33 \\
(100.0)\end{array}$ & 1 \\
\hline & Yes & $3(0.4)$ & $3(0.4)$ & $0(0.0)$ & \\
\hline \multicolumn{6}{|c|}{ Steroid use (\%) } \\
\hline & No & $\begin{array}{l}646 \\
(91.6)\end{array}$ & $\begin{array}{l}615 \\
(91.5)\end{array}$ & $\begin{array}{l}31 \\
(93.9)\end{array}$ & 1 \\
\hline & Yes & $59(8.4)$ & $57(8.5)$ & $2(6.1)$ & \\
\hline \multicolumn{6}{|c|}{ Diagnosis (\%) } \\
\hline & Lumbar disc herniation & $\begin{array}{l}176 \\
(25.0)\end{array}$ & $\begin{array}{l}168 \\
(25.0)\end{array}$ & $8(24.2)$ & 0.543 \\
\hline & Lumbar spinal stenosis & $\begin{array}{l}144 \\
(20.4)\end{array}$ & $\begin{array}{l}134 \\
(19.9)\end{array}$ & $\begin{array}{l}10 \\
(30.3)\end{array}$ & \\
\hline & $\begin{array}{l}\text { Lumbar instability/ } \\
\text { spondylolisthesis }\end{array}$ & $\begin{array}{l}169 \\
(24.0)\end{array}$ & $\begin{array}{l}162 \\
(24.1)\end{array}$ & $7(21.2)$ & \\
\hline & $\begin{array}{l}\text { Lumbar instability/ } \\
\text { spondylolisthesis combined with } \\
\text { spinal stenosis }\end{array}$ & $\begin{array}{l}216 \\
(30.6)\end{array}$ & $\begin{array}{l}208 \\
(31.0)\end{array}$ & $8(24.2)$ & \\
\hline \multirow[t]{2}{*}{ ASA (\%) } & 1 & $\begin{array}{l}250 \\
(35.5)\end{array}$ & $\begin{array}{l}242 \\
(36.0)\end{array}$ & $8(24.2)$ & 0.005 \\
\hline & II & $\begin{array}{l}327 \\
(46.4)\end{array}$ & $\begin{array}{l}316 \\
(47.0)\end{array}$ & $\begin{array}{l}11 \\
(33.3)\end{array}$ & \\
\hline
\end{tabular}

Abbreviations: $\mathrm{BMI}$, body mass index; $\mathrm{COPD}$, chronic obstructive pulmoriary disease; $\mathrm{CHF}$, chronic heart failure; ASA, American Society of Anesthesiologists; EBL, estimated blood loss; HbA1c, glycated hemoglobin A1c; Alb, albumin; RBC, red blood cells; WBC, white blood cells; PT, prothrombin time; APTT: activated partial thromboplastin time; Fib, fibrinogen. 


\begin{tabular}{|c|c|c|c|c|}
\hline & Total & $\begin{array}{l}\text { Non- } \\
\text { SSI }\end{array}$ & SSI & $\begin{array}{l}P \\
\text { value }\end{array}$ \\
\hline III & $\begin{array}{l}114 \\
(16.2)\end{array}$ & $\begin{array}{l}101 \\
(15.0)\end{array}$ & $\begin{array}{l}13 \\
(39.4)\end{array}$ & \\
\hline IV & $14(2.0)$ & $13(1.9)$ & $1(3.0)$ & \\
\hline $\begin{array}{l}\text { Operation time } \\
\text { (min) }\end{array}$ & $\begin{array}{l}155.1 \\
(26.7)\end{array}$ & $\begin{array}{l}153.2 \\
(24.5)\end{array}$ & $\begin{array}{l}193.8 \\
(37.4)\end{array}$ & $\begin{array}{l}< \\
0.001\end{array}$ \\
\hline EBL (ml) & $\begin{array}{l}209.4 \\
(63.3)\end{array}$ & $\begin{array}{l}205.1 \\
(58.4)\end{array}$ & $\begin{array}{l}296.7 \\
(91.9)\end{array}$ & $\begin{array}{l}<.001 \\
0.001\end{array}$ \\
\hline $\begin{array}{l}\text { Number of fusion } \\
\text { segments }\end{array}$ & $\begin{array}{l}1.1 \\
(0.2)\end{array}$ & $\begin{array}{l}1.1 \\
(0.2)\end{array}$ & $\begin{array}{l}1.4 \\
(0.3)\end{array}$ & $\begin{array}{l}<.001 \\
0.00\end{array}$ \\
\hline $\begin{array}{l}\text { Preoperative } \\
\text { HbA1c (\%) }\end{array}$ & $\begin{array}{l}5.4 \\
(1.0)\end{array}$ & $\begin{array}{l}5.3 \\
(0.8)\end{array}$ & $\begin{array}{l}7.5 \\
(1.6)\end{array}$ & $\begin{array}{l}< \\
0.001\end{array}$ \\
\hline $\begin{array}{l}\text { Preoperative Alb } \\
(\mathrm{g} / \mathrm{L})\end{array}$ & $\begin{array}{l}42.8 \\
(3.5)\end{array}$ & $\begin{array}{l}43.0 \\
(3.4)\end{array}$ & $\begin{array}{l}39.3 \\
(3.6)\end{array}$ & $\begin{array}{l}<.001 \\
0.001\end{array}$ \\
\hline $\begin{array}{l}\text { Preoperative } \\
\text { globulin (g/L) }\end{array}$ & $\begin{array}{l}24.1 \\
{[21.9} \\
26.3]\end{array}$ & $\begin{array}{l}24.1 \\
{[21.8} \\
26.2]\end{array}$ & $\begin{array}{l}24.6 \\
{[22.2} \\
27.5]\end{array}$ & 0.17 \\
\hline $\begin{array}{l}\text { Preoperative } \\
\text { glycated protein } \\
(\%)\end{array}$ & $\begin{array}{l}2.2 \\
(0.1)\end{array}$ & $\begin{array}{l}2.2 \\
(0.1)\end{array}$ & $\begin{array}{l}2.1 \\
(0.2)\end{array}$ & 0.001 \\
\hline $\begin{array}{l}\text { Preoperative RBC } \\
\left(* 10^{9} / \mathrm{L}\right)\end{array}$ & $\begin{array}{l}5.1[4.8, \\
5.4]\end{array}$ & $\begin{array}{l}5.1[4.9, \\
5.4]\end{array}$ & $\begin{array}{l}5.0[4.8, \\
5.1]\end{array}$ & 0.079 \\
\hline $\begin{array}{l}\text { Preoperative WBC } \\
\left({ }^{\star} 10^{9} / \mathrm{L}\right)\end{array}$ & $\begin{array}{l}7.0[6.1 \\
7.8]\end{array}$ & $\begin{array}{l}7.0[6.0, \\
7.8]\end{array}$ & $\begin{array}{l}7.4[6.7, \\
7.9]\end{array}$ & 0.07 \\
\hline $\begin{array}{l}\text { Preoperative } \\
\text { thrombocyte } \\
\left(* 10^{9} / \mathrm{L}\right)\end{array}$ & $\begin{array}{l}238.7 \\
(45.5)\end{array}$ & $\begin{array}{l}238.7 \\
(45.8)\end{array}$ & $\begin{array}{l}238.3 \\
(38.8)\end{array}$ & 0.965 \\
\hline $\begin{array}{l}\text { Preoperative PT } \\
\text { (s) }\end{array}$ & $\begin{array}{l}11.6 \\
(1.0)\end{array}$ & $\begin{array}{l}11.6 \\
(1.0)\end{array}$ & $\begin{array}{l}11.8 \\
(1.2)\end{array}$ & 0.249 \\
\hline $\begin{array}{l}\text { Preoperative } \\
\text { APTT (s) }\end{array}$ & $\begin{array}{l}31.6 \\
(1.3)\end{array}$ & $\begin{array}{l}31.6 \\
(1.2)\end{array}$ & $\begin{array}{l}31.9 \\
(2.0)\end{array}$ & 0.203 \\
\hline $\begin{array}{l}\text { Preoperative Fib } \\
\text { (mg/dL) }\end{array}$ & $\begin{array}{l}3.6 \\
(0.4)\end{array}$ & $\begin{array}{l}3.6 \\
(0.4)\end{array}$ & $\begin{array}{l}3.2 \\
(0.5)\end{array}$ & $\dot{0.001}$ \\
\hline
\end{tabular}

Abbreviations: $\mathrm{BMI}$, body mass index; $\mathrm{COPD}$, chronic obstructive pulmoriary disease; $\mathrm{CHF}$, chronic heart failure; ASA, American Society of Anesthesiologists; $\mathrm{EBL}$, estimated blood loss; HbA1c, glycated hemoglobin A1c; Alb, albumin; RBC, red blood cells; WBC, white blood cells; PT, prothrombin time; APTT: activated partial thromboplastin time; Fib, fibrinogen. 


\begin{tabular}{|c|c|c|c|c|}
\hline & Total & $\begin{array}{l}\text { Non- } \\
\text { SSI }\end{array}$ & SSI & $\begin{array}{l}P \\
\text { value }\end{array}$ \\
\hline $\begin{array}{l}\text { Preoperative } \\
\text { potassium } \\
(\mathrm{mmol} / \mathrm{L})\end{array}$ & $\begin{array}{l}4.1 \\
(0.3)\end{array}$ & $\begin{array}{l}4.1 \\
(0.3)\end{array}$ & $\begin{array}{l}4.1 \\
(0.3)\end{array}$ & 0.503 \\
\hline $\begin{array}{l}\text { Preoperative } \\
\text { calcium }(\mathrm{mmol} / \mathrm{L})\end{array}$ & $\begin{array}{l}2.4 \\
(0.1)\end{array}$ & $\begin{array}{l}2.4 \\
(0.1)\end{array}$ & $\begin{array}{l}2.4 \\
(0.1)\end{array}$ & 0.504 \\
\hline \multicolumn{5}{|c|}{$\begin{array}{l}\text { Abbreviations: BMI, body mass index; COPD, chronic obstructive pulmoriary disease; CHF, chronic } \\
\text { heart failure; ASA, American Society of Anesthesiologists; EBL, estimated blood loss; HbA1c, glycated } \\
\text { hemoglobin A1c; Alb, albumin; RBC, red blood cells; WBC, white blood cells; PT, prothrombin time; } \\
\text { APTT: activated partial thromboplastin time; Fib, fibrinogen. }\end{array}$} \\
\hline
\end{tabular}

\subsection{Univariate and multivariate logistic regression analyses of SSI}

In univariable analysis, age, $\mathrm{BMI}, \mathrm{ASA}$, operation time, $\mathrm{EBL}$, number of fusion segments, preoperative $\mathrm{HbA1c}$, preoperative Alb, preoperative glycated protein, and preoperative fibrinogen were all significantly associated with the occurrence of $\mathrm{SSI}$ in the overall population $(\mathrm{P}<0.05)$. In multivariable logistic regression analysis (Table 2), variables with statistical significance (age, BMI, ASA, operation time, EBL, number of fusion segments, preoperative $\mathrm{HbA1c}$, preoperative Alb, preoperative glycated protein, and preoperative fibrinogen) in univariate regression were included. Six variables were identified as independent risk factors for SSI, including advanced age (OR 1.187, 95\% Cl 1.006-1.401), BMI (OR 1.445, 95\% Cl 1.005-2.076), preoperative HbA1c (OR 35.390, 95\% Cl 21.215-40.124), EBL (OR 1.424, 95\% Cl 1.021-1.732), operation time (OR 1.041, 95\% Cl 1.003-1.080), and preoperative albumin (OR $0.672,95 \%$ $\mathrm{Cl} 0.463-0.975)$. 
Table 2

Univariate and multivariate logistic regression model analyses of SSI following MIS-TLIF under Quadrant channel in this study.

\begin{tabular}{|c|c|c|c|c|}
\hline & Univariate analysis & & Multivariate analysis & \\
\hline & OR $(95 \% \mathrm{Cl})$ & $\begin{array}{l}P \\
\text { value }\end{array}$ & OR $(95 \% \mathrm{Cl})$ & $\begin{array}{l}\mathrm{P} \\
\text { value }\end{array}$ \\
\hline Age (years) & $1.145(1.085-1.207)$ & $\dot{0}_{0.001}$ & $1.187(1.006-1.401)$ & 0.043 \\
\hline Sex $(\%)$ & $0.905(0.449-1.827)$ & 0.782 & NA & \\
\hline BMI (kg/m2) & $1.498(1.303-1.722)$ & $<.001$ & $1.445(1.005-2.076)$ & 0.047 \\
\hline Smoking (\%) & $3.162(1.563-6.397)$ & 0.001 & NA & \\
\hline Alcohol (\%) & $1.063(0.506-2.231)$ & 0.872 & NA & \\
\hline Diabetes (\%) & $2.450(2.175-9.104)$ & $<001$ & NA & \\
\hline COPD (\%) & $0.844(0.111-6.431)$ & 0.87 & NA & \\
\hline Stroke (\%) & $0.000(0.000-\operatorname{lnf})$ & 0.987 & NA & \\
\hline Rheumatic disease (\%) & $1.204(0.155-9.332)$ & 0.859 & NA & \\
\hline $\mathrm{CHF}(\%)$ & $0.000(0.000-\operatorname{lnf})$ & 0.988 & NA & \\
\hline Steroid use (\%) & $0.696(0.162-2.984)$ & 0.626 & NA & \\
\hline Diagnosis (\%) & $0.892(0.661-1.204)$ & 0.454 & NA & \\
\hline ASA (\%) & $1.849(1.201-2.847)$ & 0.005 & NA & \\
\hline Operation time (min) & $1.055(1.040-1.071)$ & $<.001$ & $1.041(1.003-1.080)$ & 0.035 \\
\hline $\mathrm{EBL}(\mathrm{ml})$ & $1.822(1.015-2.785)$ & $<0.001$ & $1.424(1.021-1.732)$ & 0.017 \\
\hline Number of fusion segments & $2.292(1.080-4.863)$ & 0.031 & NA & \\
\hline Preoperative HbA1c (\%) & $\begin{array}{l}11.394(5.876- \\
22.092)\end{array}$ & $<0.001$ & $\begin{array}{l}35.390(21.215- \\
40.124)\end{array}$ & $\begin{array}{l}< \\
0.001\end{array}$ \\
\hline Preoperative Alb $(\mathrm{g} / \mathrm{L})$ & $0.747(0.673-0.830)$ & $<.001$ & $0.672(0.463-0.975)$ & 0.036 \\
\hline
\end{tabular}

Abbreviations: $\mathrm{BMI}$, body mass index; $\mathrm{COPD}$, chronic obstructive pulmoriary disease; $\mathrm{CHF}$, chronic heart failure; ASA, American Society of Anesthesiologists; EBL, estimated blood loss; HbA1c, glycated hemoglobin A1c; Alb, albumin; RBC, red blood cells; WBC, white blood cells; PT, prothrombin time; APTT: activated partial thromboplastin time; Fib, fibrinogen; OR: odds ratio; $\mathrm{Cl}$, confidence interval; NA, not available. 


\begin{tabular}{|c|c|c|c|}
\hline & Univariate analysis & & Multivariate analysis \\
\hline Preoperative globulin (g/L) & $1.079(0.969-1.202)$ & 0.167 & NA \\
\hline $\begin{array}{l}\text { Preoperative glycated protein } \\
(\%)\end{array}$ & $0.010(0.001-0.168)$ & 0.001 & NA \\
\hline Preoperative RBC $\left({ }^{*} 10^{9} / \mathrm{L}\right)$ & $0.526(0.220-1.258)$ & 0.149 & NA \\
\hline Preoperative WBC $\left(* 10^{9} / \mathrm{L}\right)$ & $1.250(0.945-1.655)$ & 0.118 & NA \\
\hline $\begin{array}{l}\text { Preoperative thrombocyte } \\
\left({ }^{*} 10^{9} / \mathrm{L}\right)\end{array}$ & $1.000(0.992-1.008)$ & 0.965 & NA \\
\hline Preoperative PT (s) & $1.221(0.870-1.714)$ & 0.249 & NA \\
\hline Preoperative APTT (s) & $1.195(0.909-1.573)$ & 0.203 & NA \\
\hline Preoperative Fib (mg/dL) & $0.074(0.029-0.190)$ & $<.001$ & NA \\
\hline $\begin{array}{l}\text { Preoperative potassium } \\
\text { (mmol/L) }\end{array}$ & $0.685(0.226-2.071)$ & 0.502 & NA \\
\hline Preoperative calcium (mmol/L) & $0.358(0.018-7.247)$ & 0.503 & NA \\
\hline \multicolumn{4}{|c|}{$\begin{array}{l}\text { Abbreviations: BMI, body mass index; COPD, chronic obstructive pulmoriary disease; CHF, chronic } \\
\text { heart failure; ASA, American Society of Anesthesiologists; EBL, estimated blood loss; HbA1c, glycated } \\
\text { hemoglobin A1c;Alb, albumin; RBC, red blood cells; WBC, white blood cells; PT, prothrombin time; } \\
\text { APTT: activated partial thromboplastin time; Fib, fibrinogen; OR: odds ratio; Cl, confidence interval; NA, } \\
\text { not available. }\end{array}$} \\
\hline
\end{tabular}

\subsection{Predictive ML algorithms performance}

Comparisons of the performance of prediction among the six $\mathrm{ML}$ algorithms models in the whole set are detailed in Fig. 2. The result of the 10-fold CV of six ML algorithms in the whole set was demonstrated in Fig. 3. It turned out that the NB model demonstrated the highest performance in predicting SSI, whose average AUC was 0.86 , sensitivity 0.667 , specificity 0.89 , and accuracy 0.90 in whole sets. Accordingly, we chose the NB model as the final prediction model.

\subsection{Relative importance of variables}

According to the indicators, the NB model is determined as the final prediction model. The relative importance of variables in the NB model was shown in Fig. 4. The importance of high-ranking variables in the NB model was arranged as follows in descending order: preoperative $\mathrm{HbA1c}, \mathrm{EBL}$, operation time, preoperative Alb, BMI, and age.

\section{Discussion}


In this work, we developed and validated multiple ML algorithms to predict the risk of SSI following MISTLIF under the Quadrant channel. We found that the NB model provided improved predictive ability compared to other models. This study produced interesting findings concerning the added value of machine-learning methods, contrary to some of the wider literature on clinical prediction models. This ML-based model may potentially assist clinical decision-making when assessing and advising patients after MIS-TLIF under the Quadrant channel and when deciding on ongoing management.

MIS-TLIF is well known to have many advantages compared to open TLIF in terms of reducing muscle injury, smaller incisions, and scarring, especially for elderly patients who can not bear the trauma of traditional open TLIF surgery[20-22]. In contrast, SSI after MIS-TLIF can cause additional prolonged antibiotic use, prolonged length of stay, increased risk of fusion failure, and even the need for re-invasive surgery or removal of the implant[23,24], which not only increases the medical burden on patients and society but also causes increased mortality in patients.

With the continuous development of minimally invasive spine surgery, MIS-TLIF has gradually attracted the attention of spine surgeons. It has been reported that the incidence of SSI after lumbar interbody fusion is $2.4-5 \%[25-28]$. In this investigation, the incidence of SSI was 4.78, which was consistent with the previous report. Although risk factors associated with SSI after MIS-TLIF have been previously reported, little attention has been paid to the incidence of SSI following MIS-TLIF under the Quadrant channel. More importantly, we still lack a concise and reliable tool to identify high-risk groups and assist clinicians in their decision-making. Therefore, in this study, several ML-based models were constructed using Al techniques to predict the risk of SSI.

Many studies have shown that diabetes mellitus is an important risk factor for SSI after spine surgery[23, 29-31]. Usually, glucose metabolism is closely related to the structure and pathophysiology of blood vessels. Disorders of glucose metabolism are inextricably linked to vascular structure and dysfunction, as has been demonstrated in many studies. And vascular pathological alterations cause local microcirculatory disorders leading to tissue ischemia and hypoxia, which provide a favorable environment for the growth of anaerobic bacteria and pose a potential risk for delayed postoperative wound healing and surgical site infection. Also, systemic immune deficiency caused by diabetes is the main reason for the occurrence of SSI. Therefore, surgeons usually strictly control patients' perioperative blood glucose and control preoperative fasting glucose below $6.9 \mathrm{mmol} / \mathrm{L}$ before considering surgical treatment[32]. Interestingly, glycosylated hemoglobin has also been identified as a risk factor for SSI. Chronic hyperglycemic state leads to cell-mediated immune and macrophage dysfunction, reduced overall immune function of the body, and diminished ability to respond to bacterial viruses. And the relative importance in our prediction model was ranked first. Therefore, we need to be more cautious about the perioperative glycemic management of patients.

In the present study, we found that high BMI is also a risk factor for SSI. In a large retrospective study by Onyekwelu et al.[33], BMI > $30 \mathrm{~kg} / \mathrm{m} 2$ was found to be a high-risk factor for SSI after lumbar spinal fusion. The problems caused by obesity are present in almost all orthopedic surgeries, not only in spine 
surgery. Obesity can lead to endocrine disruption and insulin resistance, causing impaired glucose tolerance and even diabetes. On the other hand, obese patients may have a variety of underlying diseases that cause a decrease in the patient's immune capacity. Equally worrisome is the increased vascularity in the adipose tissue of obese patients and the even greater incidence of postoperative fat liquefaction, which also increases the risk of SSI.

Intriguingly, age was also identified as an independent risk factor in this study. However, we are also aware that this is a non-modifiable risk factor. Previous studies reported that the risk of SSI after spinal surgery was three times higher in patients aged $\geq 60$ years than in the younger group $[1,27]$. Similar results were observed in the present study. Although it is still controversial whether advanced age is a risk factor for SSI, the current mainstream of scholars believe that elderly patients have reduced metabolism, degenerated immune function, and may be accompanied by numerous underlying diseases, which significantly reduce the reserve capacity of the organism and make it more sensitive to surgical trauma strikes. The ability of local tissues to resist infection as well as self-repair is weakened, increasing the risk of SSI following spine surgery.

Although univariate logistics regression analysis indicated a statistical difference in operation time and EBL between the two groups $(P<0.05)$, in multivariate logistic regression analysis, we found that $E B L$ and operation time were identified as risk factors for SSI. It has been reported in the literature that prolonged operation time is a significant risk factor for SSI that can not be ignored. Prolonged operation time leads to increased tissue stretching time, aggravating local ischemia and tissue inflammatory factor release, while prolonged surgical wound exposure leads to an increased possibility of direct blood entry of pathogenic bacteria from the wound. Whereas the relationship between EBL and SSI has been debatable. According to previous literature, intraoperative blood transfusion $>1000 \mathrm{ml}$ was reported as a risk factor for postoperative SSI in spine surgery and cardiac surgery[7, 27]. Pullter et al.[33] investigated 3175 patients undergoing spine surgery and found that the incidence of postoperative SSI was $2.5 \%$ in patients with intraoperative bleeding $<1000 \mathrm{ml}$, and $3.5 \%$ in patients with intraoperative bleeding $>1000 \mathrm{ml}$. The incidence of SSI was 5.6\% ( $p=0.001)$ and was identified as an independent risk factor for SSI.

In the present study, we found that low serum albumin was an important risk factor for SSI. Serum albumin level is closely related to the nutritional status, liver and kidney function of patients. Hypoproteinemia will lead to immune suppression, local tissue edema, impaired microcirculation, and increased tissue fluid exudation, which will affect normal wound healing and create conditions for bacterial colonization. Previous literature has reported preoperative hypocalcemia as a risk factor for postoperative spinal infection[30], and another interesting finding is that electrolyte variables such as blood calcium and potassium in this investigation were not statistically significantly different between the SSI and Non-SSI groups. Patients with toxic shock syndrome are usually found to suffer from hypocalcemia, which may be due to an increase in calcitonin secretion caused by the infection, resulting in lower blood calcium levels[34]. Many previous studies have reported hypocalcemia in approximately $40 \%$ of patients with bacteremia[35]. According to the inclusion and exclusion criteria of our study, none of the patients in the 2 groups had a significant active infection before surgery, so it is not difficult to 
explain why there was no statistically significant difference in preoperative blood calcium between the 2 groups.

Compared with other research attempting to determine the risk of SSI following MIS-TLIF, our work has several advantages. First, a few studies focused on patients undergoing MIS-TLIF under the Quadrant channel. This may provide a reference for spine surgeons. Furthermore, while ML approaches have shown unparalleled prediction performance in MIS-TLIF under the Quadrant channel in recent reports, There is, however, little research in the available literature on applying ML algorithms to SSI following MIS-TLIF. As far as we know, this is the first study to develop a prediction model using ML algorithms for risk evaluation of SSI with easy-to-use clinical and laboratory data. Fortunately, our prediction model evidences good predictive performance of the model, which distinguishes itself from linear models adopted by previous research.

In this study, several limitations of this study require attention. First, the nature of a retrospective study might have introduced a selection bias. Second, the ML algorithm model we established, to some extent, was confined to one single center, which might restrict its generalizability pending further validation in real-world scenarios. Third, further independent external validation is required to confirm these findings.

\section{Conclusions}

We developed and validated ML algorithms for individualized prediction of SSI following MIS-TLIF under the Quadrant channel by utilizing readily available preoperative variables. The ML-based prediction model can identify whether patients are at high risk of SSI and may assist the clinician in decision making. In the future, we hope to enlarge the sample size in the following study and obtain more reliable achievements further.

\section{Abbreviations}

BMI: body mass index; COPD: chronic obstructive pulmoriary disease; CHF: chronic heart failure; ASA: American Society of Anesthesiologists; EBL: estimated blood loss; HbA1c: glycated hemoglobin A1c; Alb: albumin; RBC: red blood cells; WBC: white blood cells; PT: prothrombin time; APTT: activated partial thromboplastin time; Fib: fibrinogen; MIS-TLIF: minimally invasive transforaminal lumbar interbody fusion; CV: cross-validation; KNN: k-Nearest Neighbor; DT: Decision tree; SVM: Support vector machine; RF: Random Forest; MLP: Multi-Layer Perceptron; NB: Naïve Bayes; ACC: accuracy; AUC: area under the receiver operating characteristic curve; $\mathrm{CV}$ : cross-validation; TP: true positive; TN: true negative; FP: false positive; FN: false negative; OR: odds ratio; $\mathrm{Cl}$ : confidence interval; $\mathrm{ML}$ : machine learning; Al: artificial intelligence; EMR, electronic medical record.

\section{Declarations}

\section{Ethics approval and consent to participate}


The study was approved by an institutional ethics committee at the Taizhou Central Hospital (Affiliated Hospital to Taizhou College). Considering that this work was a retrospective study: the ethics committee waived the requirement for informed consent from patients.

\section{Consent for publication}

Not applicable.

\section{Availability of data and materials}

The data set supporting the conclusion of this article is available on request to the corresponding author.

\section{Competing interests}

The authors declare that they have no competing interests.

\section{Funding}

No funds were received in support of this work.

\section{Authors' contributions}

HSW collected the data, analyzed the data, and drafted the manuscript. MYY supervised the project and reviewed the manuscript. BY, WLL, TTF, and QL conceived of the study, participated in its design and coordination, and helped to draft the manuscript. MYY was responsible for the whole project, designed the study, and supervised the study. All authors read and approved the final manuscript.

\section{Acknowledgements}

Not applicable.

\section{References}

1. Lee W-c, Park J-Y, Kim KH, Kuh SU, Chin DK, Kim KS, Cho YE: Minimally invasive transforaminal lumbar interbody fusion in multilevel: comparison with conventional transforaminal interbody fusion. World neurosurgery 2016, 85:236-243.

2. Park Y, Ha JW, Lee YT, Sung NY: Minimally invasive transforaminal lumbar interbody fusion for spondylolisthesis and degenerative spondylosis: 5-year results. Clinical Orthopaedics and Related Research $\circledast 2$ 2014, 472(6):1813-1823.

3. Park Y, Seok S-O, Lee S-B, Ha J-W: Minimally invasive lumbar spinal fusion is more effective than open fusion: a meta-analysis. Yonsei medical journal 2018, 59(4):524-538.

4. Kulkarni $A G$, Patel RS, Dutta S: Does minimally invasive spine surgery minimize surgical site infections? Asian spine journal 2016, 10(6):1000. 
5. O'Toole JE, Eichholz KM, Fessler RG: Surgical site infection rates after minimally invasive spinal surgery. Journal of Neurosurgery: Spine 2009, 11(4):471-476.

6. Akins PT, Harris J, Alvarez JL, Chen Y, Paxton EW, Bernbeck J, Guppy KH: Risk factors associated with 30-day readmissions after instrumented spine surgery in 14,939 patients. Spine 2015, 40(13):1022-1032.

7. Kim BD, Smith TR, Lim S, Cybulski GR, Kim JY: Predictors of unplanned readmission in patients undergoing lumbar decompression: multi-institutional analysis of 7016 patients. Journal of Neurosurgery: Spine 2014, 20(6):606-616.

8. Adogwa O, Elsamadicy AA, Han JL, Karikari IO, Cheng J, Bagley CA: 30-day readmission after spine surgery: an analysis of 1400 consecutive spine surgery patients. Spine 2017, 42(7):520-524.

9. Bai Z, Hu S, Wang Y, Deng W, Gu N, Zhao R, Zhang W, Ma Y, Wang Z, Liu Z et al: Development of a machine learning model to predict the risk of late cardiogenic shock in patients with ST-segment elevation myocardial infarction. Ann Trans/ Med 2021, 9(14):1162.

10. Hochman E, Feldman B, Weizman A, Krivoy A, Gur S, Barzilay E, Gabay H, Levy J, Levinkron O, Lawrence G: Development and validation of a machine learning-based postpartum depression prediction model: A nationwide cohort study. Depress Anxiety 2021, 38(4):400-411.

11. Pollock KG, Sekelj S, Johnston E, Sandler B, Hill NR, Ng FS, Khan S, Nassar A, Farooqui U: Application of a machine learning algorithm for detection of atrial fibrillation in secondary care. Int J Cardiol Heart Vasc 2020, 31:100674.

12. Qin H, Hu X, Zhang J, Dai H, He Y, Zhao Z, Yang J, Xu Z, Hu X, Chen Z: Machine-learning radiomics to predict early recurrence in perihilar cholangiocarcinoma after curative resection. Liver Int 2021, 41(4):837-850.

13. Segal Z, Kalifa D, Radinsky K, Ehrenberg B, Elad G, Maor G, Lewis M, Tibi M, Korn L, Koren G: Machine learning algorithm for early detection of end-stage renal disease. BMC Nephro/ 2020, 21(1):518.

14. Mangram AJ, Horan TC, Pearson ML, Silver LC, Jarvis WR, Committee HICPA: Guideline for prevention of surgical site infection, 1999. Infection Control \& Hospital Epidemiology 1999, 20(4):247-280.

15. Shin S, Austin PC, Ross HJ, Abdel-Qadir H, Freitas C, Tomlinson G, Chicco D, Mahendiran M, Lawler $\mathrm{PR}$, Billia $\mathrm{F}$ et al: Machine learning vs. conventional statistical models for predicting heart failure readmission and mortality. ESC Heart Fail 2021, 8(1):106-115.

16. Siu BMK, Kwak GH, Ling L, Hui P: Predicting the need for intubation in the first $\mathbf{2 4} \mathbf{~}$ after critical care admission using machine learning approaches. Sci Rep 2020, 10(1):20931.

17. SOFO L: New perspectives in the prediction of postoperative complications for high-risk ulcerative colitis patients: machine learning preliminary approach. 2020.

18. Tewari P, Kashdan E, Walsh C, Martin CM, Parnell AC, O'Leary JJ: Estimating the conditional probability of developing human papilloma virus related oropharyngeal cancer by combining machine learning and inverse Bayesian modelling. PLoS Comput Biol 2021, 17(8):e1009289. 
19. Pedregosa F, Varoquaux G, Gramfort A, Michel V, Thirion B, Grisel O, Blondel M, Prettenhofer P, Weiss $\mathrm{R}$, Dubourg V: Scikit-learn: Machine learning in Python. the Journal of machine Learning research 2011, 12:2825-2830.

20. Kim J-S, Jung B, Lee S-H: Instrumented minimally invasive spinal-transforaminal lumbar interbody fusion (MIS-TLIF). Clinical spine surgery 2018, 31(6):E302-E309.

21. Lee $\mathrm{KH}$, Yeo W, Soeharno H, Yue WM: Learning curve of a complex surgical technique: minimally invasive transforaminal lumbar interbody fusion (MIS TLIF). Clinical Spine Surgery 2014, 27(7):E234E240.

22. Tan JH, Liu G, Ng R, Kumar N, Wong H-K: Is MIS-TLIF superior to open TLIF in obese patients?: a systematic review and meta-analysis. European Spine Journal 2018, 27(8):1877-1886.

23. Gum JL, Carreon LY, Stimac JD, Glassman SD: Predictors of Oswestry Disability Index worsening after lumbar fusion. Orthopedics 2013, 36(4):e478-e483.

24. Hart RA, Cabalo A, Bess S, Akbarnia BA, Boachie-Adjei O, Burton D, Cunningham ME, Gupta M, Hostin $\mathrm{R}$, Kebaish $\mathrm{K}$ : Comparison of patient and surgeon perceptions of adverse events after adult spinal deformity surgery. Spine 2013, 38(9):732-736.

25. Lim S, Edelstein Al, Patel AA, Kim BD, Kim J: Risk factors for postoperative infections after singlelevel lumbar fusion surgery. Spine 2018, 43(3):215-222.

26. Liu J-M, Deng H-L, Chen X-Y, Zhou Y, Yang D, Duan M-S, Huang S-H, Liu Z-L: Risk factors for surgical site infection after posterior lumbar spinal surgery. Spine 2018, 43(10):732-737.

27. Sebastian A, Huddleston III P, Kakar S, Habermann E, Wagie A, Nassr A: Risk factors for surgical site infection after posterior cervical spine surgery: an analysis of 5,441 patients from the ACS NSQIP 2005-2012. The Spine Journal 2016, 16(4):504-509.

28. Veeravagu A, Patil CG, Lad SP, Boakye M: Risk factors for postoperative spinal wound infections after spinal decompression and fusion surgeries. Spine 2009, 34(17):1869-1872.

29. Deng H, Chan AK, Ammanuel SG, Chan AY, Oh T, Skrehot HC, Edwards CS, Kondapavulur S, Nichols $A D$, Liu C: Risk factors for deep surgical site infection following thoracolumbar spinal surgery. Journal of Neurosurgery: Spine 2019, 32(2):292-301.

30. Klemencsics I, Lazary A, Szoverfi Z, Bozsodi A, Eltes P, Varga PP: Risk factors for surgical site infection in elective routine degenerative lumbar surgeries. The Spine Journa/ 2016, 16(11):13771383.

31. Yao R, Zhou H, Choma TJ, Kwon BK, Street J: Surgical site infection in spine surgery: who is at risk? Global spine journal 2018, 8(4_suppl):5S-30S.

32. Olsen MA, Nepple JJ, Riew KD, Lenke LG, Bridwell KH, Mayfield J, Fraser VJ: Risk factors for surgical site infection following orthopaedic spinal operations. JBJS 2008, 90(1):62-69.

33. Onyekwelu I, Glassman SD, Asher AL, Shaffrey Cl, Mummaneni PV, Carreon LY: Impact of obesity on complications and outcomes: a comparison of fusion and nonfusion lumbar spine surgery. Journal of Neurosurgery: Spine 2017, 26(2):158-162. 
34. Aderka D, Schwartz D, Dan M, Levo Y: Bacteremic hypocalcemia: A comparison between the calcium levels of bacteremic and nonbacteremic patients with infection. Archives of internal medicine 1987, 147(2):232-236.

35. Chesney RW, McCarron D, Haddad J, Hawker C, DiBella F, Chesney P, Davis J: Pathogenic mechanisms of the hypocalcemia of the staphylococcal toxic-shock syndrome. The Journal of laboratory and clinical medicine 1983, 101(4):576-585.

\section{Figures}

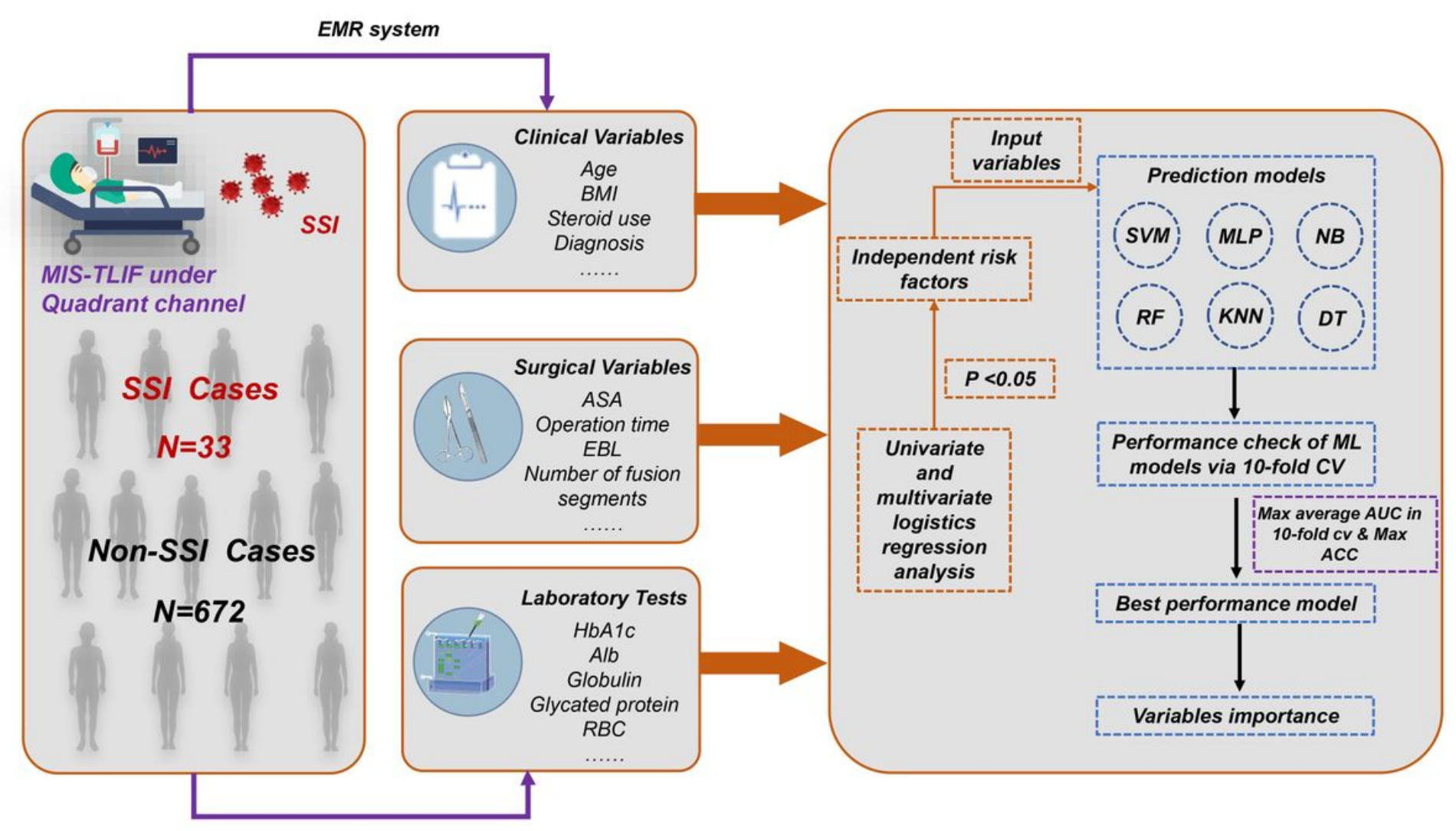

EMR system

\section{Figure 1}

Overall flowchart of whole process. The final prediction model was determined by the result of maximum AUC and ACC. MIS-TLIF, minimally invasive transforaminal lumbar interbody fusion; SSI, surgical site infection; EMR: electronic medical record; AUC: area under the curve; ACC: accuracy; KNN, k-Nearest Neighbor; DT, Decision tree; SVM, Support vector machine; RF, Random Forest; MLP, Multi-Layer Perceptron; NB, Naïve bayes. 
A

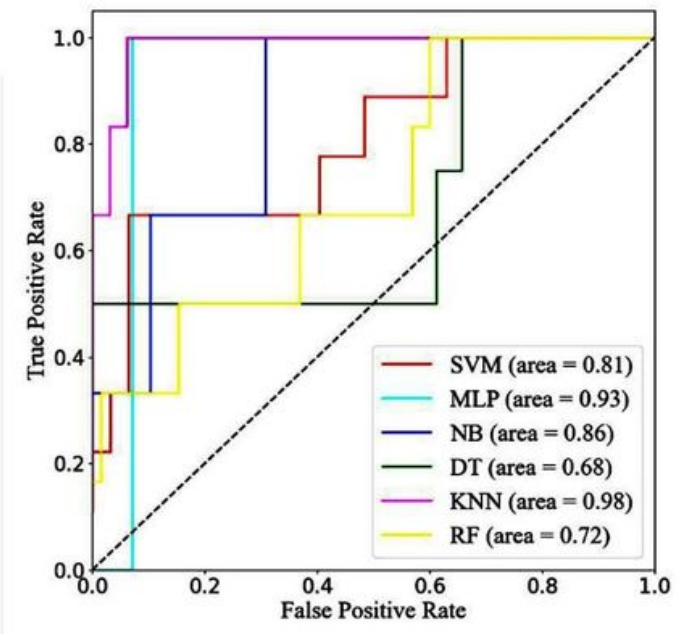

B

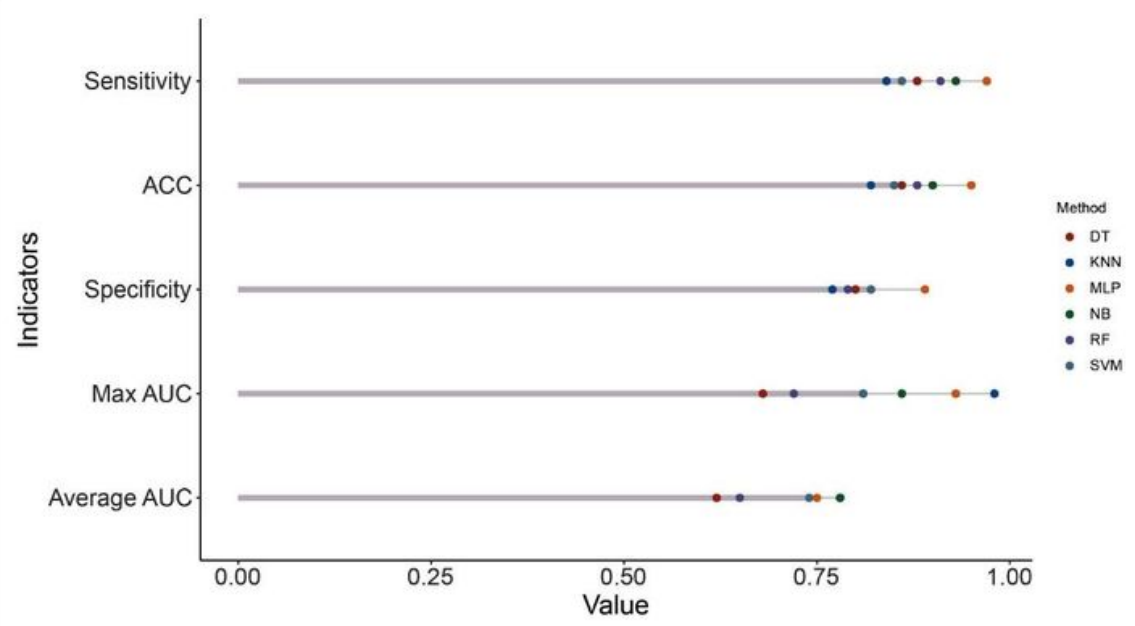

\section{Figure 2}

a. ROC curve analysis of machine learning algorithms for predicting the risk of SSI following MIS-TLIF under Quadrant channel in total set. b. Predictive performance comparison of the six types of machine learning algorithms in the total set. SSI, surgical site infection; MIS-TLIF, minimally invasive transforaminal lumbar interbody fusion; ROC, Receiver Operating Characteristic curve; KNN, k-Nearest Neighbor; DT, Decision tree; SVM, Support vector machine; RF, Random Forest; MLP, Multi-Layer Perceptron; NB, Naïve bayes. 


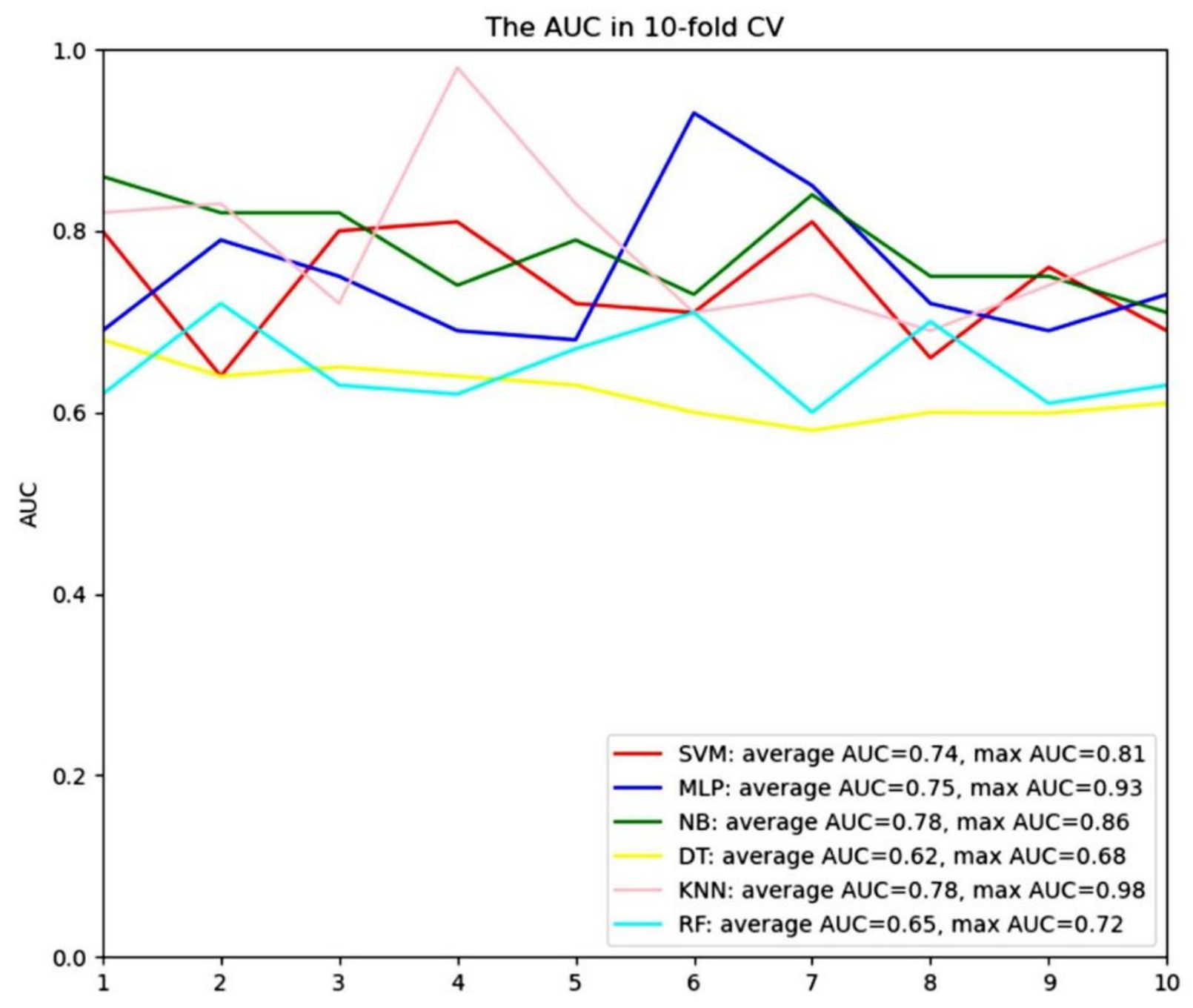

Figure 3

ROC curve analysis of a 10 -fold CV of six machine learning algorithms for predicting the risk of surgical site infection following minimally invasive transforaminal lumbar interbody fusion under Quadrant channel in total set. CV, cross validation; KNN, k-Nearest Neighbor; DT, Decision tree; SVM, Support vector machine; RF, Random Forest; MLP, Multi-Layer Perceptron; NB, Naïve bayes. 


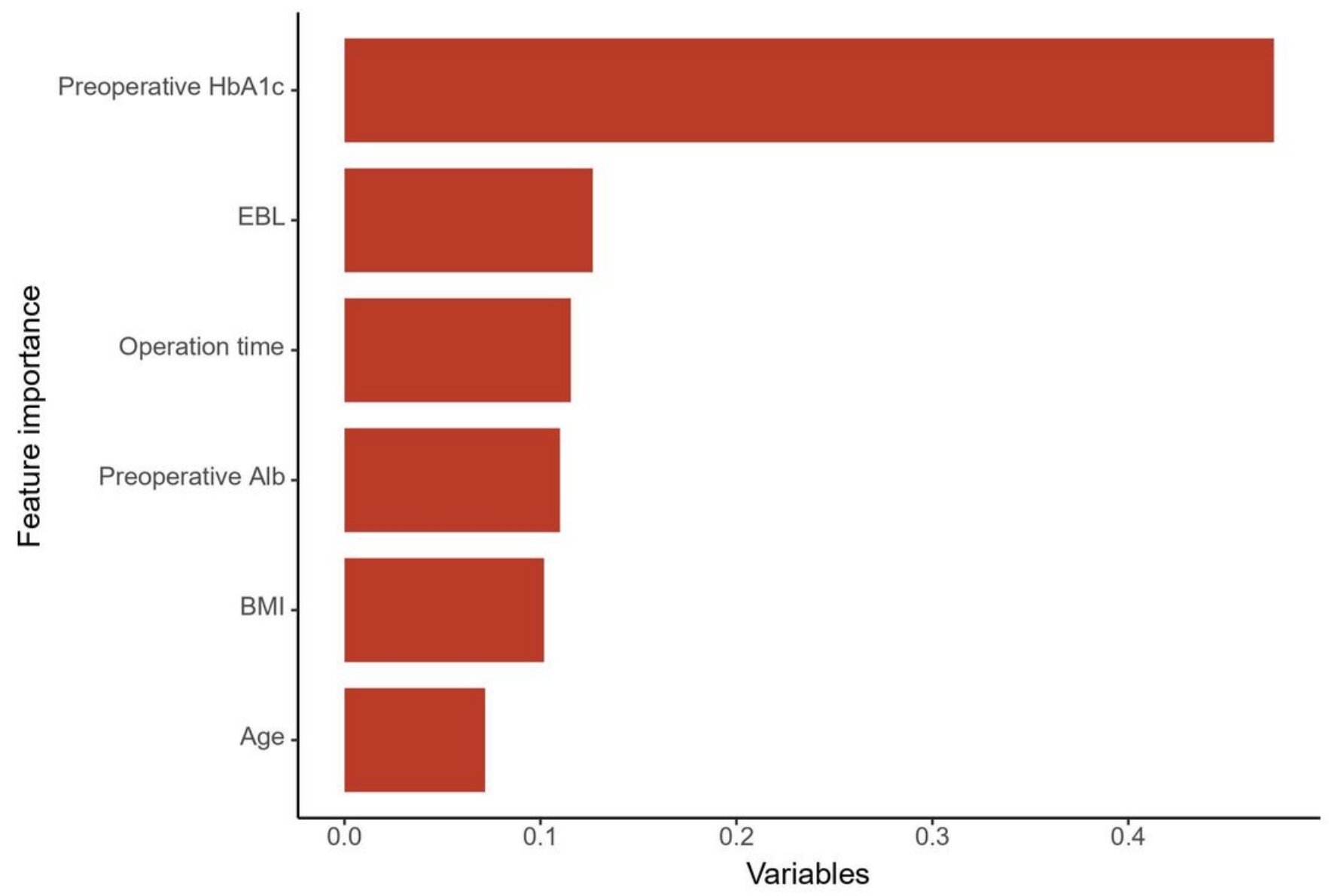

\section{Figure 4}

The importance of the variables in the Multi-Layer Perceptron model is in decreasing order as follows: Preoperative $\mathrm{HbA1c}$, EBL, operation time, preoperative Alb, BMI, and age. HbA1c, glycated hemoglobin A1c; EBL, estimated blood loss; Alb, albumin; BMI, body mass index. 